und sichert hiermit die Entscheidung des Schuldners darüber, ob derartiges Staatseigentum verwertet werden kann, ohne dass hierfür die Gewährung „freien Geleits" im Sinne des § 20 Kulturgut-Abwanderungsschutzgesetz erforderlich ist. Die entsprechende Anwendung des $\S 882$ a Abs. 2 ZPO ist auch geboten, da es sich vorliegend um zum nationalen Kulturgut des betroffenen Staates gehörende Kunstgegenstände handelt.
Die Kostenentscheidung beruht auf § 91 Abs. 1 ZPO.

Die Streitwertfestsetzung richtet sich nach $\S 53$ Abs. 1 Nr. 1 GKG iVm § 3 ZPO. (Entscheidung von der Redaktion bearbeitet.)

\title{
Kunsthandwerk und künstlerische Tätigkeit - Zur Definition des Kunstbe- griffs im Sinne des Künstlersozialversicherungsgesetzes (KSVG)
}

\author{
Sozialgericht Bremen, Urteil vom 25. März 2010 - S 4 KR 77/07
}

\section{Bei der Herstellung von Ausschneidebogen für den Bau mechanischer Skulpturen aus Papier handelt es sich um eine künstlerische Tätigkeit iSd Künstlersozialversicherungsgesetzes (KSVG). Da die Ausschneidebogen das Ergebnis einer eigenschöpferischen Leistung sind, bei dem der Schwerpunkt auf der Idee der zu erzählenden Ge- schichte sowie der gestalterischen Umsetzung liegt, handelt es sich nicht um eine Form von (Kunst-) Handwerk. 2. Trotz der handwerklichen Arbeit ist eine Zuordnung zum Bereich der Kunst im Übrigen dann möglich, wenn der handwerklich tätige Künstler mit seinen Werken in Kunstkreisen als Künstler anerkannt und behandelt wird. 3. Die Möglichkeit, eine Idee theoretisch unbegrenzt zu reproduzieren, schließt Kunst nicht aus. (Leitsätze der Redaktion)}

\section{Tenor}

Unter Aufhebung des Bescheides der Beklagten vom 28. August 2006 und des Widerspruchsbescheides vom 11. Mai 2007 wird festgestellt, dass der Kläger seit dem 1. Januar 2007 der Versicherungspflicht nach dem Künstlersozialversicherungsgesetz unterliegt. Im Übrigen wird die Klage abgewiesen. Die außergerichtlichen Kosten des Klägers hat die Beklagte zu erstatten.

\section{Tatbestand}

- Streitig ist die Versicherungspflicht des Klägers nach dem Künstlersozialversicherungsgesetz (KSVG).

Der 1949 geborene Kläger ist ausgebildeter Lehrer mit beiden Staatsexamina. Bis Ende März 2004 arbeitete er insgesamt ca. 25 Jahre in der beruflichen Weiterbildung. Bereits Ende November 2000 meldete der Kläger daneben ein Gewerbe für die Herstellung und den Verkauf von Skulpturen sowie die Herstellung und den Verkauf von mechanischen Modellen an. Nach der Kündigung durch seinen Arbeitgeber bezog der Kläger im Zeitraum 1. April 2004 bis 25. Mai 2006 Arbeitslosengeld I. Ausweislich des Einkommenssteuerbescheides 2006 erzielte er im Kalenderjahr 2006 1.247,00 Euro an Einkünften aus Gewerbebetrieb.

Am 17. Mai 2006 stellte der Kläger bei der Beklagten den Antrag auf Feststellung der Versicherungspflicht. Er sei nach Auslaufen seines Arbeitslosengeldes von nun [an] als Maler, Zeichner, künstlerischer Grafiker, Bildhauer sowie mit ähnlicher künstlerischer Tätigkeit im Bereich bildender Kunst hauptberuflich selbstständig tätig. Die von ihm entwickelten und aus
Papier bestehenden mechanischen Modelle verkaufe er als Ausschneidebogen über das Internet weltweit sowie über lokale Geschäftspartner und Zwischenhändler. Die Bausätze seien teilweise auch in Fachgeschäften und Museumsshops erhältlich. Daneben fertige er insbesondere für Museen Einzelstücke und Großskulpturen als Auftragsarbeit, organisiere Ausstellungen durch seine Papiermechanik-Sammlung, führe Papiermechanik-Workshops als VHS-Kurse und im Rahmen der Lehrerfortbildung durch und habe im Jahr 2003 ein englischsprachiges Handbuch über Papiermaschinen veröffentlicht sowie mehrere Fachartikel verfasst.

Auf die Bitte der Beklagten im Verwaltungsverfahren, Nachweise über die Anerkennung als Künstler in entsprechenden Fachkreisen bildender Künstler vorzulegen, teilte der Kläger mit, der Anerkennungsnachweis sei schwierig zu erbringen. Sein Hauptprodukt "Ausschneidebogen für den Bau mechanischer Skulpturen" sei in der deutschen akademischen Kunstszene weitgehend unbekannt. International sei dies anders. Dort sei er als Künstler anerkannt.

Mit Bescheid vom 28. August 2006 lehnte die Beklagte die Feststellung der Versicherungspflicht nach dem KSVG ab. Bei der Tätigkeit des Klägers handele es sich um Kunsthandwerk. Der Kläger fertige Skulpturen aus Pappe an. Um eine künstlerische Tätigkeit handele es sich nur dann, wenn eine entsprechende Anerkennung in einschlägigen fachkundigen Kreisen als Künstler anerkannt sei. Dafür sei hier nichts ersichtlich.

Gegen den ablehnenden Bescheid der Beklagten legte der Kläger mit Schreiben vom 13. September 2006 Widerspruch ein. Es handele sich nicht um eine handwerkliche, sondern um eine 
grafische Tätigkeit. Er entwerfe Ausschneidebogen. Dies sei das Ergebnis einer geistig-kreativen Anstrengung. Idee, Konzept, Skizze, Entwurf, Zeichnung der einzelnen Bauteile, Kolorierung sowie Zeichnung der Bauanleitung auf der Basis isometrischer Grafiken sei die Tätigkeit eines Grafikdesigners. Zudem habe er seine Arbeiten in zahlreichen Museen präsentiert. Auch dies spreche für die erforderliche Anerkennung als Künstler.

Mit Widerspruchsbescheid vom 11. Mai 2007 wies die Beklagte den Widerspruch als unbegründet zurück. Die Tätigkeit des Klägers beschränke sich auf die Herstellung „allgemeiner Bastelbögen“. Eine Gleichstellung mit Bildhauerkunst sei nicht gerechtfertigt. Eine Anerkennung als Künstler sei nicht dargelegt.

Am 31. Mai 2007 hat der Kläger Klage erhoben. Er betont, dass die Tätigkeit sich durch einen hohen grafisch-designerischen Anteil auszeichne. Konsequenterweise sei er Mitglied der Allianz Deutscher Designer (AGD). Zudem ergebe sich insbesondere aus seinen Großskulpturen, dass es sich um der Bildhauerkunst gleichgestellte Kunstobjekte handele und nicht um Spielzeug. Zudem illustriere er für Zeitschriften (u.a. für ein Geolino-Extraheft) und habe mit verschiedenen Verlagen Illustrationsverträge abgeschlossen. An Ausstellungen nehme er zum Beispiel an einer (internationalen) Antikriegsausstellung teil. Im Jahr 2006 sei er noch Berufsanfänger gewesen, denn zuvor habe er seine Tätigkeit nur nebenberuflich ausgeübt.

\section{Er beantragt,}

den Bescheid der Beklagten vom 28. August 2006 und den Widerspruchsbescheid vom 11. Mai 2007 aufzuheben und festzustellen, dass er seit dem 26. Mai 2006 der Versicherungspflicht nach dem Künstlersozialversicherungsgesetz unterliegt.

Die Beklagte beantragt,

die Klage abzuweisen.

Sie verweist zunächst darauf, dass aufgrund der Betriebsgründung im Jahr 2000 zum Zeitpunkt der Antragstellung 2006 das Berufsanfängerprivileg nicht mehr gegolten habe. Im Kalenderjahr 2006 sei ausweislich des Einkommenssteuerbescheides aber die Mindesteinkommensgrenze nach § 3 KSVG nicht erreicht worden. Aber auch in der Sache selbst liege keine künstlerische Tätigkeit im Sinne des KSVG vor. Insbesondere sei die Entwicklung mechanischer Ausschneidebogen, mit denen der Kläger nach eigenen Angaben seine Haupteinkünfte erziele, nicht als Schaffen bildender Kunst einzustufen. Vielmehr bestehe die Tätigkeit in der Entwicklung von Bastelutensilien, die der sinnvollen Freizeitgestaltung dienlich seien. Daneben sei der Kläger auch kein Grafik- oder Produktdesigner. Denn Grafikdesigner würden grafische Kommunikationsmittel wie Anzeigen, Firmenlogos, Broschüren oder Internetauftritte entwerfen. Produktdesigner beschäftigten sich dagegen mit der angepassten Gestaltung von Gebrauchsgegenständen aller Art. Design setze aber immer voraus, dass sich die Tätigkeit auf den Entwurf beschränke. Auch handele es sich nicht um Bildhauerkunst. Denn deren Werke zeichneten sich gerade dadurch aus, dass mit ihnen kein weitergehender Zweck verbunden werde. Anders sei es mit den Großarbeiten des Klägers, die museumspädagogischen Zwecken dienten. Im Übrigen spreche die Ausstellung in Sachmuseen nicht für eine Anerkennung im Bereich bildender Künstler.

Das Gericht hat Stellungnahmen von Herrn Dr. D. sowie von Herrn C. eingeholt, auf deren Inhalt an dieser Stelle verwiesen wird. Im Rahmen der mündlichen Verhandlung hat sich die Kammer drei der - zusammengebauten - Skulpturen vom Kläger vorführen lassen. Wegen der weiteren Einzelheiten wird auf den Inhalt der beigezogenen Verwaltungsvorgänge der Beklagten sowie den Inhalt der gewechselten Schriftsätze verwiesen.

\section{Entscheidungsgründe}

Die als kombinierte Anfechtungs- und Feststellungsklage nach $\S 55$ Abs. 1 Sozialgerichtsgesetz (SGG) statthafte Klage ist überwiegend begründet. Die angegriffenen Bescheide sind rechtswidrig und verletzen den Kläger in seinen Rechten. Denn seit dem Kalenderjahr 2007 besteht für ihn als selbstständigen Künstler Versicherungspflicht nach dem KSVG.

Rechtsgrundlage des geltend gemachten Feststellungsanspruchs ist $\S 1 \mathrm{iVm} \S 2$ Satz 1 KSVG. Nach $\S 1$ KSVG sind selbständige Künstler und Publizisten in der allgemeinen Rentenversicherung, in der gesetzlichen Krankenversicherung und in der sozialen Pflegeversicherung pflichtversichert, wenn sie die künstlerische oder publizistische Tätigkeit erwerbsmäßig und nicht nur vorübergehend ausüben und im Zusammenhang mit der künstlerischen oder publizistischen Tätigkeit nicht mehr als einen Arbeitnehmer beschäftigen, es sei denn, die Beschäftigung erfolgt zur Berufsausbildung oder ist geringfügig im Sinne des § 8 des Vierten Buches Sozialgesetzbuch. Nach § 2 KSVG ist Künstler im Sinne des Gesetzes, wer Musik, darstellende oder bildende Kunst schafft, ausübt oder lehrt. Publizist im Sinne dieses Gesetzes ist, wer als Schriftsteller, Journalist oder in anderer Weise publizistisch tätig ist oder Publizistik lehrt.

In § 2 Satz 1 KSVG werden drei Bereiche künstlerischer Tätigkeit jeweils in den Spielarten des Schaffens, Ausübens und Lehrens umschrieben, nämlich die Musik sowie die bildende und die darstellende Kunst. Eine weitergehende Festlegung, was darunter im Einzelnen zu verstehen ist, ist im Hinblick auf die Vielfalt, Komplexität und Dynamik der Erscheinungsformen künstlerischer Betätigungsfelder nicht erfolgt. Der Gesetzgeber spricht im KSVG nur allgemein von „Künstlern“ und „künstlerischen Tätigkeiten", auf eine materielle Definition des Kunstbegriffs hat er hingegen bewusst verzichtet (BT-Drucks 8/3172, S. 21). Dieser Begriff ist deshalb aus dem Regelungszweck des KSVG unter Berücksichtigung der allgemeinen Verkehrsauffassung und der historischen Entwicklung zu erschließen (vgl. nur BSG SozR 4-5425 § 24 Nr. 6 Rn. 13 und BSGE 83, 160, $161=$ SozR 3-5425 § 2 Nr. 9 S. 33 - jeweils mwN; vgl. auch BT-Drucks 9/26, S. 18 und BT-Drucks 8/3172, S. 19 ff.). Aus den Materiali- 
en zum KSVG ergibt sich, dass der Begriff der Kunst trotz seiner Unschärfe auf jeden Fall solche künstlerischen Tätigkeiten umfassen soll, mit denen sich der „Bericht der Bundesregierung über die wirtschaftliche und soziale Lage der künstlerischen Berufe (Künstlerbericht)" aus dem Jahre 1975 (BT-Drucks 7/3071) beschäftigt (BSGE 83, 160, 165 f. = SozR 3-5425 § 2 Nr. 9 S. 37 f.; BSGE 83, 246, 250 = SozR 3-5425 § 1 Nr. 5 S. 23). Der Gesetzgeber hat damit einen an der Typologie von Ausübungsformen orientierten Kunstbegriff vorgegeben, der in aller Regel dann erfüllt ist, wenn das zu beurteilende Werk den Gattungsanforderungen eines bestimmten Kunsttyps (z.B. Theater, Malerei, Musik) entspricht. Bei diesen Berufsfeldern ist das soziale Schutzbedürfnis der Betroffenen zu unterstellen, ohne dass es auf die Qualität der künstlerischen Tätigkeit ankommt oder eine bestimmte Werk- und Gestaltungshöhe vorausgesetzt wird (BSG, aaO).

Unter Berücksichtigung der allgemeinen Verkehrsauffassung übt der Kläger eine künstlerische Tätigkeit im Sinne des KSVG aus, indem er bildende Kunst schafft. Entscheidend ist dabei auf die Herstellung der Ausschneidebogen abzustellen, weil entsprechend seiner Selbsteinschätzung, die er auf gerichtliche Nachfrage in der mündlichen Verhandlung noch einmal bestätigt hat, die Erlöse aus dem Verkauf dieser Bogen nach wie vor den Schwerpunkt seiner Einkünfte ausmachen und nicht etwa die Einnahmen aus den anscheinend zunehmenden Illustratorenverträgen mit Buchverlagen (vgl. zur Notwendigkeit der Schwerpunktbildung bei "gemischten Tätigkeiten“ BSG, Urt. v. 01. Oktober 2009 - B 3 KS 3/08 R - kreativer Tanz; Urt. v. 01. Oktober 2009 - B 3 KS 2/08 R - Vor-MFE; Urt. v. 23. März 2006 - B 3 KR 9/05 R - Trauerredner; grdl. Urt. v. 16. April 1998 - B 3 KR 7/97 R-).

Bereits typologisch und auf der Grundlage des vom BSG immer wieder herangezogenen „Künstlerberichts“ kann die Tätigkeit des Klägers als künstlerisch eingestuft werden. In Betracht kommt insbesondere eine Gleichsetzung mit bzw. Bezeichnung als künstlerischer Grafiker, als „Objektemacher" bzw. als Illustrator (vgl. zu allen BT-Drucks. 7/3071, S. 7). Insbesondere zeichnen sich die Arbeiten des Klägers durch die - zur Erfüllung des Kunstbegriffs immer erforderliche - eigenschöpferische Leistung aus (vgl. nur BSG, Urt. v. 07. Juli 2005 - B 3 KR 37/04 $\mathrm{R}-\mathrm{mwN}$ ). Denn durch das zusätzliche Element der Bewegung und damit der zeitlichen Komponente liegt der Schwerpunkt der Tätigkeit in dem kreativen Prozess, sich "eine Geschichte" auszudenken, die sodann gestalterisch und "kinetisch" umgesetzt wird. Demzufolge betont der Kläger zu Recht, dass es sich bei seinen Objekten nicht um realitätsgetreue Miniaturmodelle, sondern um „Originale im Format 1:1" handelt.

Der Beklagten ist zuzugestehen, dass eine Einordnung der Tätigkeit des Künstlers als künstlerisch Schwierigkeiten bereitet, wenn alleine auf die anhand einer historischen Entwicklung geformte Verkehrsanschauung abgestellt wird. Denn die von dem Kläger ausgeübte Form kinetischer Kunst ist - im historischen Vergleich - noch jung und gerade in der Bundesrepublik noch wenig verbreitet. Hinzu kommen mit dem Werkstoff Papier und der Reproduzierbarkeit über die Ausschneidebogen Besonderheiten der von dem Kläger hergestellten „automata“, die - wie Herr C. als Autor des Buches „Mechanische Spielobjekte und Automaten“ in seiner Stellungnahme vom 10. Mai 2009 noch einmal bestätigt hat - sie auf der einen Seite zwar besonders charakterisieren, ihre Einordnung aus Sicht des KSVG aber auch erschweren. Der Kunstbegriff des KSVG ist aber bewusst offen gehalten; sowohl gegenüber neuen Entwicklungen, als auch gegenüber Kunstformen, die, von ihrer rein quantitativen $\mathrm{Er}$ scheinung, marginal erscheinen (vgl. BSG, Urt. v. 15. November 2007 - B 3 KS 3/07 R - Kalligraf). Dabei ist insbesondere unerheblich, ob die zu beurteilende Kunstgattung Ursprünge im Ausland oder fremdländische Wurzeln besitzt (BSG, aaO).

Da die Ausschneidebogen - wie bereits beschrieben - das Ergebnis einer eigenschöpferischen Leistung sind, bei dem der Schwerpunkt auf der Idee der zu erzählenden Geschichte sowie der gestalterischen Umsetzung liegt, handelt es sich entgegen der Ansicht der Beklagten nicht um eine Form von (Kunst-) Handwerk (vgl. zur Abgrenzung von Handwerk und Kunst z.B. BSG, aaO, Urt. v. 28. Februar 2007 - B 3 KS 2/07 R - Tätowierer; Urt. v. 25. September 2001 - B 3 KR 18/00 R - Restaurator; Urt. v. 24. Juni 1998 - B 3 KR 13/97 R - Feintäschner). Dem steht bereits entgegen, dass die manuell-technischen Fertigkeiten, die die handwerkliche Tätigkeit erfordert, in Form des Zusammenbauens von den Käufern zu leisten sind, während sich der Kläger mit seiner künstlerischen Arbeit auf das Gestalterische beschränkt. Im Übrigen entspricht es der ständigen Rechtsprechung des Bundessozialgerichts, dass trotz der handwerklichen Arbeit nach eigenen Entwürfen eine Zuordnung zum Bereich der Kunst dann möglich ist, wenn der handwerklich tätige Künstler mit seinen Werken in Kunstkreisen als Künstler anerkannt und behandelt wird (vgl. nur BSG, Urt. v. 28. Februar 2007 - B 3 KS $2 / 07 \mathrm{R}$ - Tätowierer mwN). Auch im Falle des Klägers liegen insbesondere mit seiner Teilnahme an einer ganzen Reihe von Ausstellungen (vgl. nur die Ausstellung zurzeit im Südtiroler Bergbaumuseum mit dem Titel "A. - Faszination Papiermechanik" sowie die in der Akte befindlichen Ausstellungsübersichten) Indizien vor, die auf seine Anerkennung als Künstler schließen lassen.

Das Gericht nimmt an, dass die Bedenken der Beklagten, den Kläger als Künstler anzuerkennen, insbesondere mit der theoretisch unbegrenzten Reproduzierbarkeit seiner Papierobjekte zusammenhängen. Auch aus wirtschaftlicher Sicht ist die einem Objekt zugrunde liegende Idee damit in einem anderen Maße nutzbar, als wenn sie für ein Unikat „verbraucht" würde. Dies spricht hier aber nicht gegen eine künstlerische Tätigkeit im Sinne des KSVG, auch wenn der Kläger dadurch eventuell sozial weniger schutzbedürftig ist. Denn zum Einen hat die Möglichkeit, eine Idee zu reproduzieren, Kunst bisher nicht ausgeschlossen (z.B. bei Fotografen), noch tut sie dies - angesichts eines rapiden technischen Fortschritts - in der Gegenwart (z.B. bei Webdesignern). Zum Anderen hat das Gericht den Eindruck gewonnen, dass das Konzept der mit den Ausschneidebogen einhergehenden Reproduzierbarkeit gerade wesentlicher Be- 
standteil der Kunst des Klägers und damit eine Art "Markenzeichen" ist. Denn sie wird dadurch erschwinglich und verfügt über eine Breitenwirkung, die anderen als Unikat hergestellten mechanischen Objekten nicht zukommt.

Zuletzt ist darauf hinzuweisen, dass Versicherungspflicht erst ab dem Kalenderjahr 2007 vorliegt. Denn im Kalenderjahr 2006 bestand Versicherungsfreiheit nach § 3 Abs. 1 KSVG idF des Gesetzes v. 13. Juni 2001 (BGBI. I S. 1027), weil der Kläger die Mindesteinkommensgrenze nicht erreicht hat. Ihn im Jahr 2006 noch als Berufsanfänger im Sinne von § 3 Abs. 2 KSVG anzusehen, überzeugt das Gericht vor dem Hintergrund des bereits lange angemeldeten Gewerbes und der vorhergehenden Arbeitslosigkeit nicht. Anderenfalls hätten es die Betroffenen in der Hand, den Zeitpunkt des Beginns der 3-Jahres-Frist praktisch selbst zu bestimmen. Seit 2007 verfügt der Kläger demgegenüber über ein Einkommen, das die Einkommensgrenze des $\S 3$ Abs. 1 KSVG übersteigt.

Die Kostenentscheidung beruht auf $\S 193$ SGG. Sie entspricht der Billigkeit, weil die Klage ganz überwiegend erfolgreich war. Dabei hat das Gericht auch berücksichtigt, dass die Feststellung des Bestehens der Versicherungspflicht über den Tag der Urteilsverkündung hinausreicht und demgemäß der Zeitraum, in dem entgegen der Ansicht des Klägers keine Versicherungspflicht bestand, nicht mehr erheblich erscheint. (Entscheidung von der Redaktion bearbeitet.)

\title{
Varia
}

\section{Sammler, Mäzene und Kunsthändler als Wegbereiter der Moderne in Berlin 1880 - 1933}

\author{
Eine gemeinsame Konferenz des Moses Mendelssohn Zentrums für europäisch-jüdische \\ Studien, des Kollegiums Jüdische Studien an der Humboldt-Universität zu Berlin und der \\ Stiftung Brandenburger Tor vom 14. bis 16. Juni 2010 in Berlin.
}

- Die unter wissenschaftlicher Leitung von Julius Schoeps abgehaltene Konferenz hatte sich zum Ziel gesetzt, die enge Verknüpfung insbesondere deutsch-jüdischer Künstler, Kunsthändler und Sammler im Berlin der Zeit nach der Gründung des Deutschen Reichs bis zur Machtübernahme durch die Nationalsozialisten zu beleuchten und deren Bedeutung für die Etablierung der damaligen modernen Kunst herauszuarbeiten. Thematisch gegliedert war die Konferenz in fünf Themenblöcke: Vordenker und Wegbereiter, Die ersten Sammlungen, Vermittler, öffentliches und privates Mäzenatentum und Verleger als Kunstförderer. Es überrascht nicht, dass der Titel der Veranstaltung bei den mit Fragen der Provenienzforschung befassten Einrichtungen in besonderem Maße Interesse hervorrief. Dementsprechend sachkundig zeigte sich der Teilnehmerkreis.

Unter dem Titel „Mit dem nötigen Quantum Phantasie" stellte Monika Tatzkow den Künstler und Sammler Max Liebermann als einen Wegbereiter der Moderne vor, der bereits in den 1890er Jahren damit begonnen hatte, französische Impressionisten zu sammeln und ihre Verbreitung durch großzügige Leihgaben zu fördern. Mit ihrem Titel knüpfte Tatzkow dabei an eine Äußerung Lieber- manns an, der einmal festgestellt hatte, dass das nötige Quantum Phantasie aus dem Handwerk ein Kunstwerk entstehen lässt.

Christian Kennert charakterisierte am Beispiel Paul Cassirers den Impressionismus als eine geistige Bewegung.

Der in Fachkreisen mit Spannung erwartete Vortrag von Ottfried Dascher über den Kunsthändler und -sammler Alfred Flechtheim veranschaulichte den Aufstieg und bereits im Jahr 1929 einsetzenden Niedergang der von Flechtheim geführten Galerien. Auch das nicht immer leicht auseinanderzuhaltende $\mathrm{Ne}$ ben- und Miteinander des Kunsthändlers Flechtheim mit dem Kunstsammler Flechtheim versuchte Dascher auszuloten. Dascher schloss damit, dass Flechtheim zur Rettung seiner Galerie auch seine Privatsammlung verwerten musste. Insgesamt überraschte Dascher mit bislang nicht bekannten Neuigkeiten. Seine These, Flechtheims Ehefrau Betty wäre dem angesichts der unmittelbar drohenden Deportation gewählten (Frei-)Tod entkommen, wenn eine bestimmte Person, deren Namen Dascher allerdings nicht nennen wollte, weiterhin ihre beschützende Hand über sie gehalten hätte, hat zwar durchaus dramatischen, nicht aber wissenschaftlich fundierten Gehalt.
Sammler, Mäzene und Kunsthändler als Wegbereiter der Moderne in Berlin 1880 - 1933

Berlin, 14. bis 16. Juni 2010 